\title{
Technological solutions to compensate humanmeteorological measurement errors with measuring human activity and movement
}

\author{
Ádám Pintér \\ MOGI Department \\ Budapest University of Technology and Economics \\ Budapest, Hungary \\ adam.pinter@icicom.hu
}

\author{
Krisztián Samu \\ MOGI Department \\ Budapest University of Technology and Economics \\ Budapest, Hungary \\ samuk@mogi.bme.hu
}

\begin{abstract}
To enhance the objectivity of humanmeteorological measurements, various instrumental measurements are required. These equipments are suitable to explore the desired relationships, only if those are able to record simultaneously the individual's physiological and micro-environmental parameters and their transients. It is also important to possibly filter or measure the disturbances effecting our measurements in order to compensate errors in our results. In our case this factor is the physical activity of the individual, which has effect on physiological parameters, therefore we have to register its value in function of time. The following paper deals with technical and theoretical questions of measuring this factor and summarizes the ascertainment we collected during the experiments. The topic is an integral part of teh $\mathrm{PhD}$ research Á. Pintér behooves at Budapest University of Technology and Economics.
\end{abstract}

Keywords-sampling rate, acceleration measurement, human movement, humanmeteorology

\section{STATE OF THE ART}

About the topic of humanmeteorological ${ }^{\circledR}$ measurements which means measuring and exploring the effects of weather on human organism - we have taken a survey in our last article [1]. The conclusion was: However there are few articles deal with the weather's influence on health and mortality, there were not found any equipment measuring physiological signals together with the personal microenvironment in order to learn the direct relationships [2-9]. The other topic is the measurement of the physical activity. There are several articles in scientific journals about human movements. For instance measurements with tri-axis accelerometer in connection with visual inspection from video recordings [10], and often there are articles about studying human movements to improve inverse kinematics [11]. Beside these there are papers about different technical methods of measuring (view-based, acceleration-based) [12-13], but during our research we have not fount any article about measuring the human activity in order to compensate other measurement errors.

\section{INTRODUCTION}

Statements involved in this paper are related to an equipment introduced in one of our previous publications [1]. To measure objectively the atmospheric environment's effects on human organism, we developed an apparatus, which is able to record simultaneously the individual's heart signals and micro-environmental parameters, such as temperature and air pressure. For the appropriate evaluation of collected data, it is also needed to filter other factors' effects which have to be treated as disturbances. This paper does not cover the trait of the measured physiological parameters, nor the mode of evaluation of those, but instead it involves the technical improvements of the cited equipment, which intended to serve on the one hand the easier and more expedient use of the device, and on the other hand the more suitability for measuring the wearer's activity.

\section{NOISES, ERRORS}

We regard the human organism as a physical-technical MIMO (Multiply Input Multiply Output) system, where the outputs are the electric signals of the heart, but at the same time the inputs are more diverse. Since our research intended the aim to define the effects of atmospheric parameters, the ideal would be, if we could measure these effects clearly, differentiated. However, this is not possible. Even a measurement - not physiological - performed between laboratory circumstances is loaded with random errors, measure errors and the incomplete knowledge of plurality of input parameters.

The load error types and their characteristics [14]: 
- Gross, salient error: Error stem from malfunction of the measure instrument or from inattention. The origin should be investigated, and the cause should be eliminated if possible.

- Systematic error: We call systematic errors those, which are constant during repeated measurements, or which variation is pre-determinable. Causes may be known or unknown. This kind of error can arise, for example, from the operation of members of the measuring system (measuring chain) and from the operation of the measurement process as well. The sign and magnitude on the whole measurement range is known.

- Random error: A measurement error that varies randomly at the repeated measurements of the physical value. Random error is a variate. There are statistical methods that can be used to determine an error bound for the random errors.

Against a part of these we can protect by trying to built the measure equipment in such a way that it measures the parameters most accurate and most reproducibly. In our paper [1] we also discussed, that the best - in metrology often applied - way to eliminate disturbances caused by uncontrollable, external effects is the numerous, long-term measurement method, which can suppress the random noise. But there are also known, disturbing factors as well, which effects are significantly present. Unfortunately, this does not mean that we can handle all of them, but some of them. In this case this is the physical activity of the wearer. Over the condition of the nerve system and the organism, the heart's operation is highly influenced by the extent and the transients or our activity. A measure could lead to false results, for instance, when we the individual is running after an autobus or sleeping discomposedly, which significantly changes the measured parameters. In such cases we could diagnose false relationships between the supposed inputs and measured outputs. Thus, we can see, in our case it is a systematic error, assuming that we know the level of the activity in an acceptable degree regarding to the measured physiological parameters. Thus, we can significantly compensate its effect in the results about our activitymeasurements.

\section{MOVEMENT, ACTIVITY}

Movements on human body can be very diverse. In our study the exact form of the movement is not interesting, only the extent of the influence on heart operation is. In first approximation the intensity of the movement per time unit is interesting for us and the duration of the movement. We simply call it activity. We can get information about this most easily, if we register the acceleration of the individual's center of mass with a tri-axis MEMS accelerometer, we produce a vector from the three acceleration value and we integrate its absolute value over the time.
This is appropriate to separate well from each other, for instance, running after the a bus, walking on the street, working at the office and sleeping. But it does not give us sufficient information, because it matters that the quantity of the movement is based on forces by the human body - like running - or, for example result of traveling on a vehicle. Thus, it is important to make a second, more accurate approximation, where we can draw conclusions about the origin of the movement about the patterns of the movement. In the third approximation we add the information to these, which day period we are measuring the values. Than we have a time-like comparison base, that characterize the day period's typical movement pattern and extent, and then we can recognize the movements, which differs from these, which can be called "irregular" movements. At night, during sleeping even a walk-like amount of movement is irregular in the sense, that the subject individual probably woke up and went out to the bathroom. This means that the individual's physiological parameters should be treated differently.

\section{MEASUREMENTS}

In the following paragraph we illustrate the separated movements about our measurements and their measure modes. The first questions are, how, with what frequency and within what range we should measure the human activity in order to get the more information from the results. The problem is also important, because all measurements of the sensor requires electric power, which we want to economize with, as it is a mobile equipment. Data storage question is not negligible question either. In case of too much measurements, the amount of data we have to store could become huge, moreover it could unnecessarily make the data processing difficult.

Therefore we have to determine a measure frequency and strategy which characterize the human body's movement properly, but does not load the power supply more than needed. For this we worked out a method, which only starts to record the acceleration values, when the absolute acceleration exceeds a threshold. This was determined that sleeping and working in one place do not exceed this level. This value has been empirically determined with preliminary measurements. In order to simplify the design, such a MEMS sensor was chosen which possess an interrupt output with the ability of observing the acceleration in IDLE - low consumption - mode, and it is able to send a trigger signal to the microcontroller unit, when exceeds a threshold we set. In such a case the microcontroller wakes up from its IDLE mode and send a command to the sensor unit to carry out a tri-axis acceleration measurement, and the controller stores it to a memory card completed with time stamps. When the acceleration values permanently reduce under a specified level, the system goes back to IDLE mode again. To define the sampling frequency in a case of relevant activity, we used Shannon's (Nyquist) sampling theorem as a base [14]. 
Theorem: If the sampling frequency

$$
\omega_{\mathrm{s}}=\frac{2 \cdot \pi}{\mathrm{h}}
$$

is greater than two times the continuous signal's highest, essential frequency $\omega_{0}$ component,

$$
\omega_{\mathrm{s}}>2 \cdot \omega_{0} \text {, }
$$

then the original signal can be reconstructed from the sampled signal. Using the Nyquist frequency, the sampling must satisfy the following inequality:

$$
\omega_{0}<\frac{\omega_{\mathrm{s}}}{2} \Rightarrow \omega_{0}<\omega_{\mathrm{N}} \text {. }
$$

Physical quantities and their units in the formulas:

- $\omega_{\mathrm{s}}$ sampling angular frequency

$[\mathrm{Hz}]$

- $\mathrm{h}$ sampling interval

$[\mathrm{s}]$

- $\omega_{0}$ useful signal's maximum frequency $[\mathrm{Hz}]$;

- $\omega_{\mathrm{N}}$ Nyquist angular frequency

$[\mathrm{Hz}]$.

Nevertheless it determines the minimum criteria. Imagine a case, when we would like to sample a sine signal and then reconstruct it. As long as we miserably sample just at the zero-crossings, we get a constant zero signal instead of the real form of the original. Thus, it is subservient to sample eight-ten times the frequency of the movement pattern's adducent frequency. For the base of our tests we chose the maximum power running of a human, which is one of the most active and fastest movement in common days. Then the acceleration values in direction " $\mathrm{z}$ " - about experiences can be approximated very grossly with a sine-like wave with a $3[\mathrm{~Hz}]$ frequency. On a numerous experiment's results, analysing the curves we came the conclusion, that a $25[\mathrm{~Hz}]$ sampling is proper to reconstruct the signal. This confirms our theoretical starting point as well. On the following figures we can see well, that the $12.5[\mathrm{~Hz}]$ sampling frequency could not characterize well enough the pattern of human running, it was only able to represent the acceleration of the human body distorted. In turn, the sampling with $50[\mathrm{~Hz}]$ frequency war not significantly better than the $25[\mathrm{~Hz}]$, therefore in the end we decided at the $25[\mathrm{~Hz}]$ sampling rate. On the figures values in direction "z" are around $1[\mathrm{~g}]$ because of Earth's gravity acceleration.

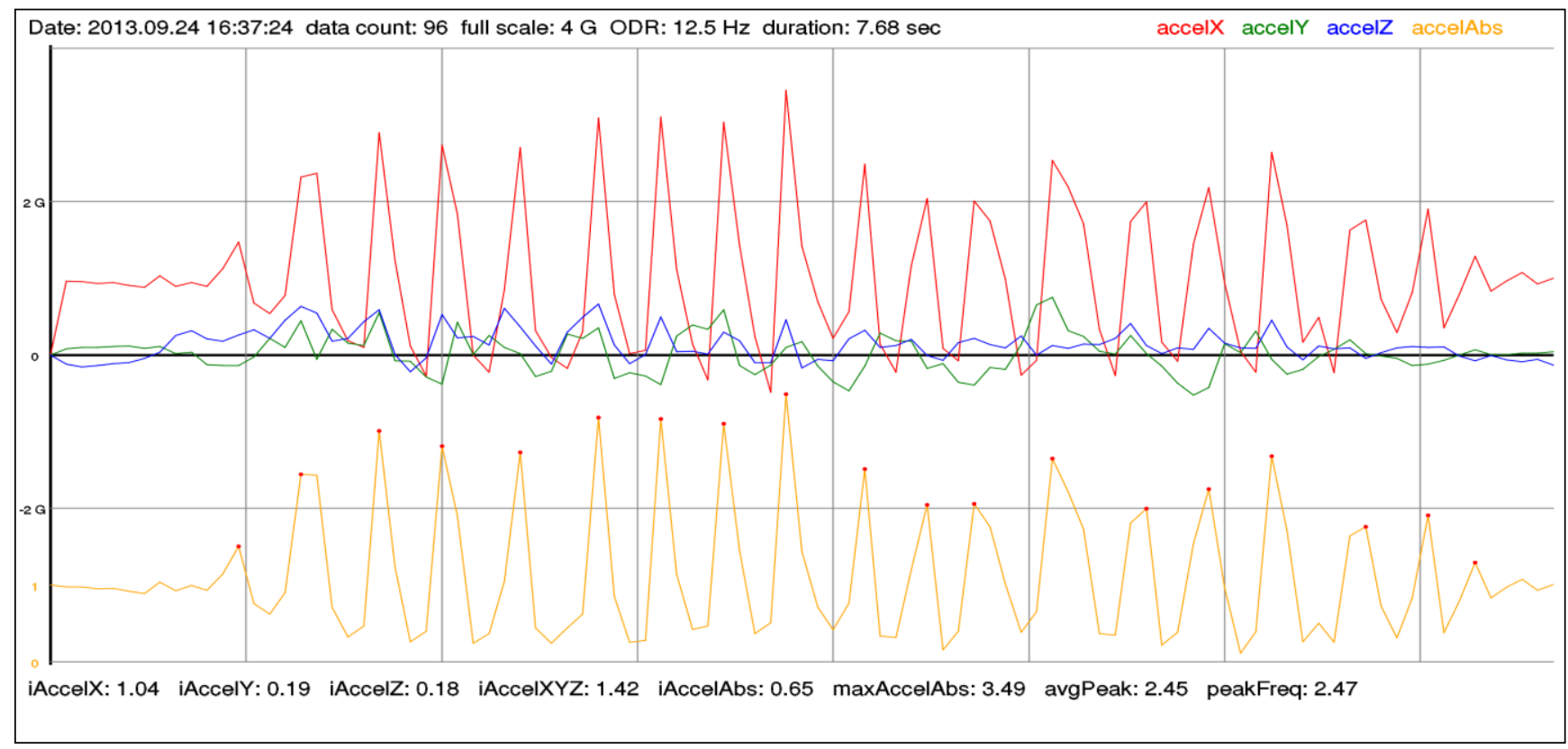

Fig. 1.: Measured acceleration values with 12.5 [Hz] sampling frequency 


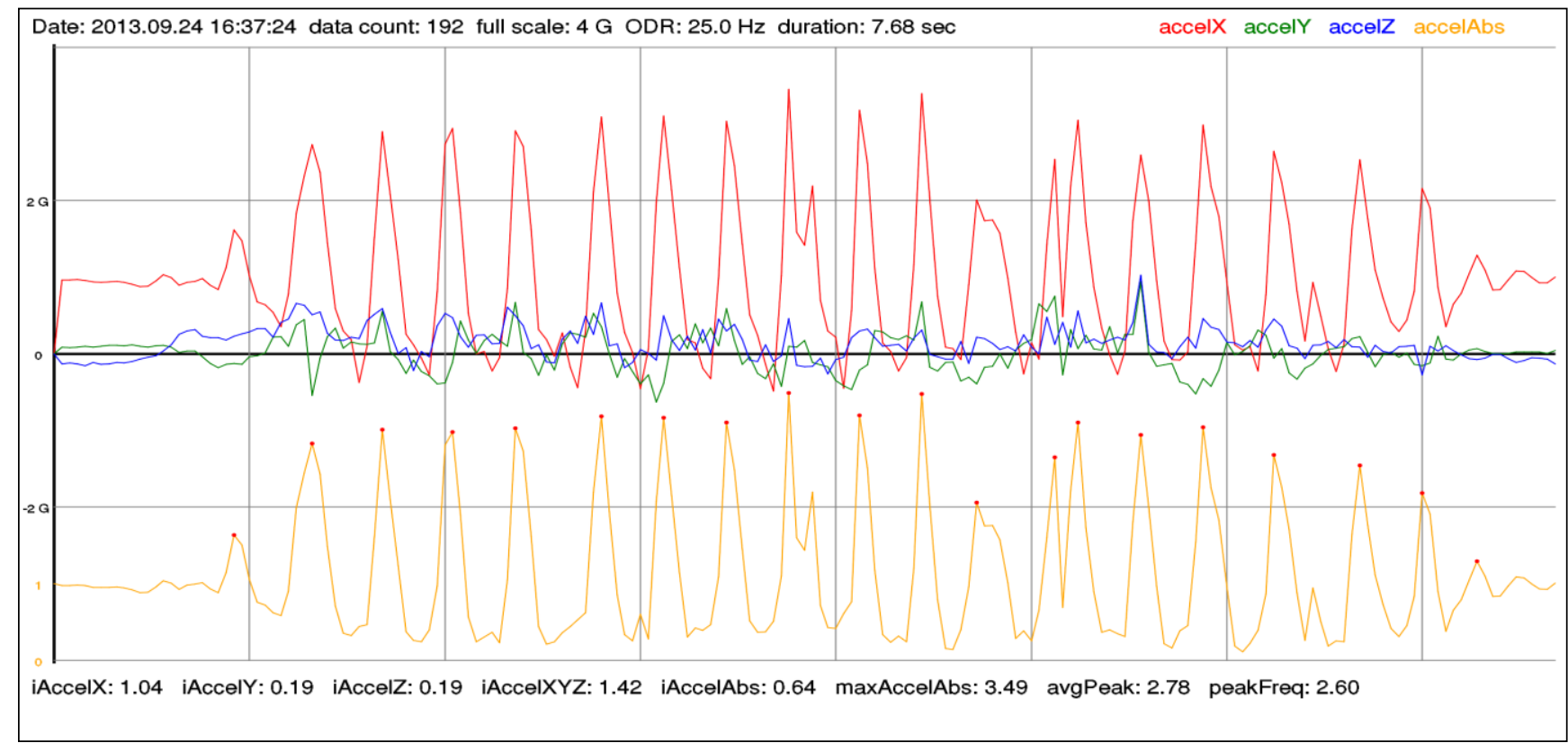

Fig. 2.: Measured acceleration values with $25[\mathrm{~Hz}]$ sampling frequency

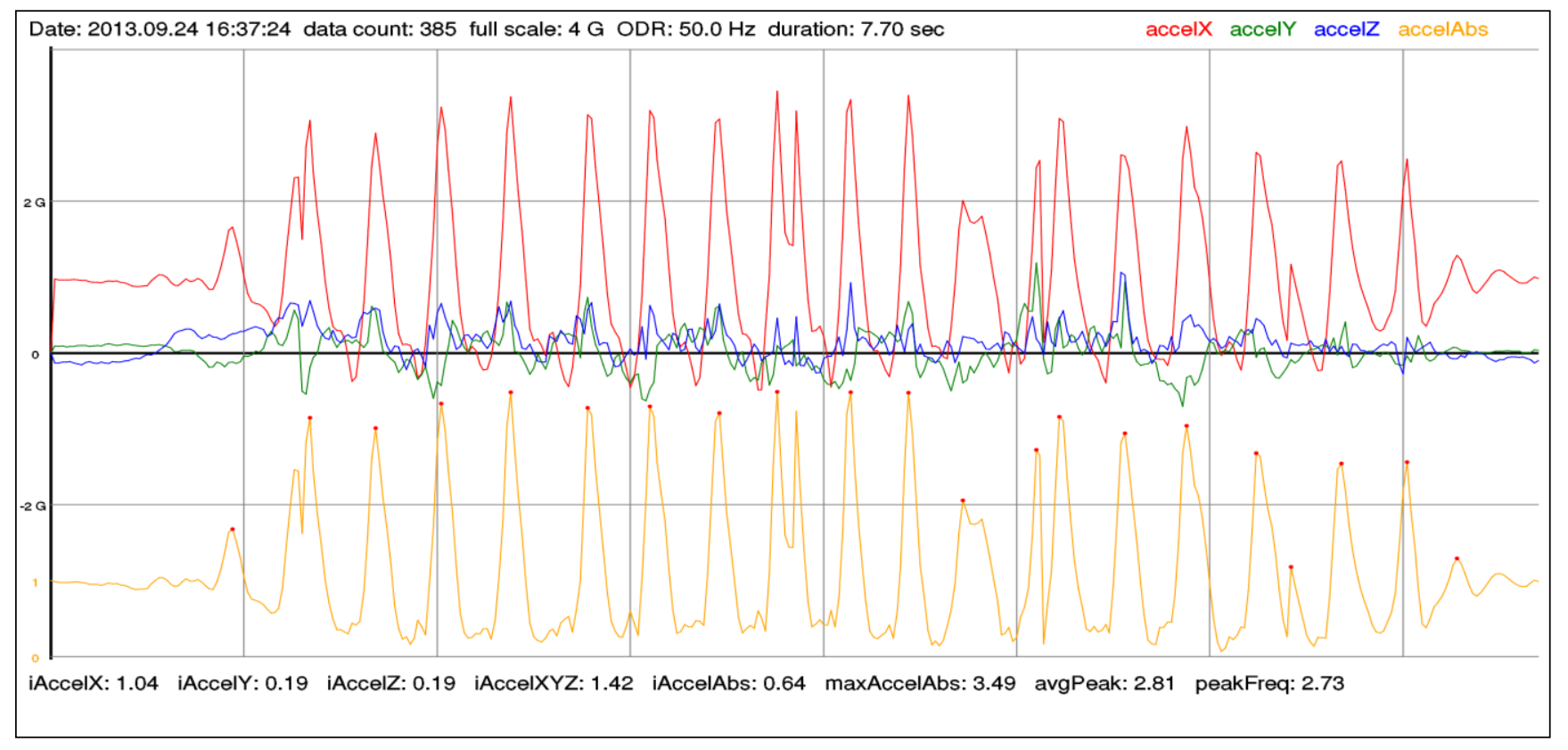

Fig. 3.: Measured acceleration values with $50[\mathrm{~Hz}]$ sampling frequency

We also have to tell a word about the measuring range. In general, MEMS accelerometers can be configured to several different measuring range. These are typically $\pm 2[\mathrm{~g}], \pm 4$ [g], and $\pm 8[\mathrm{~g}]$, or sometimes different. As long as the integrated $\mathrm{AD}$ converter encodes the full set measuring range on the same number of bits, in particular case we can achieve a poor resolution. General measurement principle is to select a measurement range, that surely includes the maximum values of the useful signal, but at the same time we should choose the narrowest of these. Consider the event that, say, if we want to register movements with $\pm 1.5[\mathrm{~g}]$ maximum acceleration, and we measure on $\pm 8[\mathrm{~g}]$ range. The given 10-bit resolution covers the $\pm 8[\mathrm{~g}]$ range anyway, however our measurements never reach this level. Accordingly, the highest bits will always be zero, does not carry information, while the finer acceleration values are under the resolution and lost. Thus we unnecessarily reduced resolution of the sensor. Since the human body is capable of producing a wide range of accelerations, we decided to work out a dynamic strategy for this question: 
- The sensor in default is in IDLE mode. It observes (as mentioned above), if the absolute acceleration exceeds the $1\left[\mathrm{~m} / \mathrm{s}^{2}\right]$ threshold. It equals $0.1[\mathrm{~g}]$.

- If yes, the sensor in default starts to measure on \pm 2 [g] with 25 [Hz] sampling frequency. In this range, for example, can fit turning in the middle of the night and going the bathroom.

- If the sensor measures a value which is close to the limit of range, it set to next, greater measure range, which is \pm 4 [g]. In this range fit most ordinary life occurring acceleration.
- If the measured data still crosses the threshold of $\pm 4 \quad[\mathrm{~g}]$, the sensor switches to the largest measurement range, $\pm 8[\mathrm{~g}]$. It is an extreme case.

- With the reduction of acceleration values, the sensor gradually returns to smaller and smaller measurement ranges, and will finally re-enters IDLE mode.

Examination of movements are continuously taking place, the figures below represents some typical movements. On figures values in direction "z" are around $1[\mathrm{~g}]$ because of Earth's gravity acceleration.

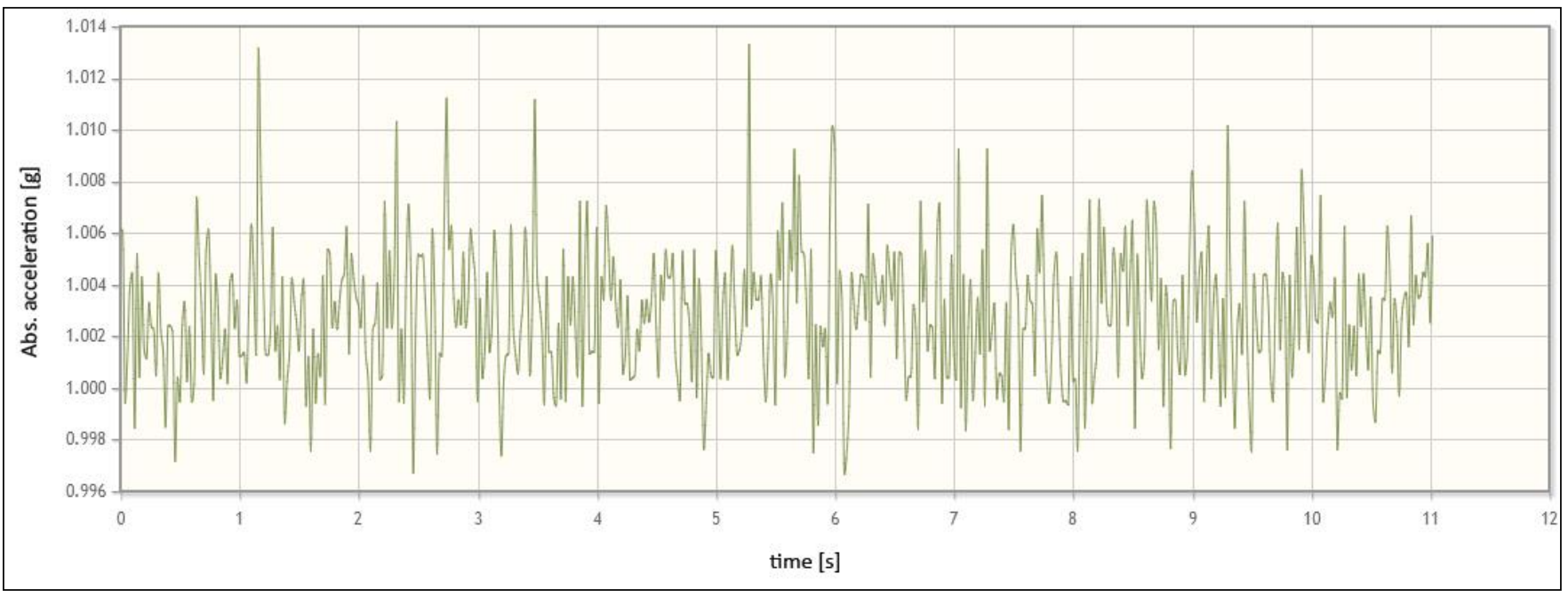

Fig. 4.: Movement pattern of sleeping in calm

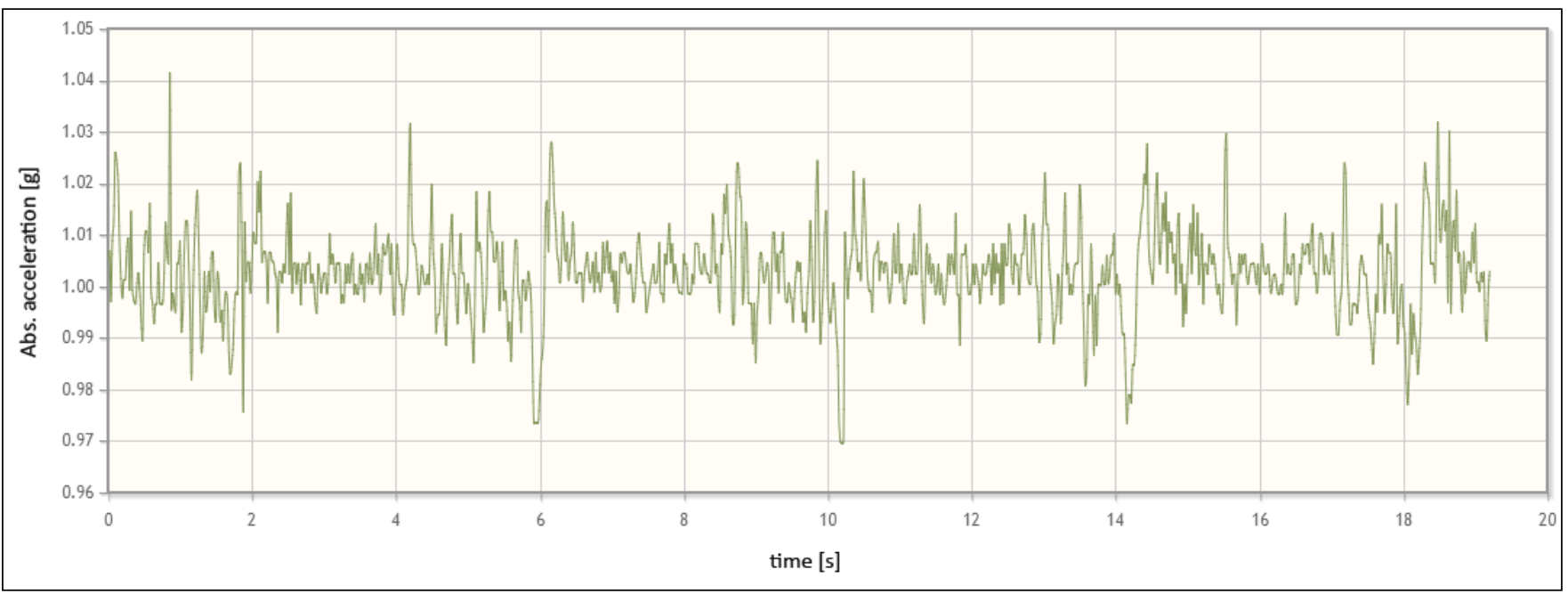

Fig. 5.: Movement pattern of awake breathing 


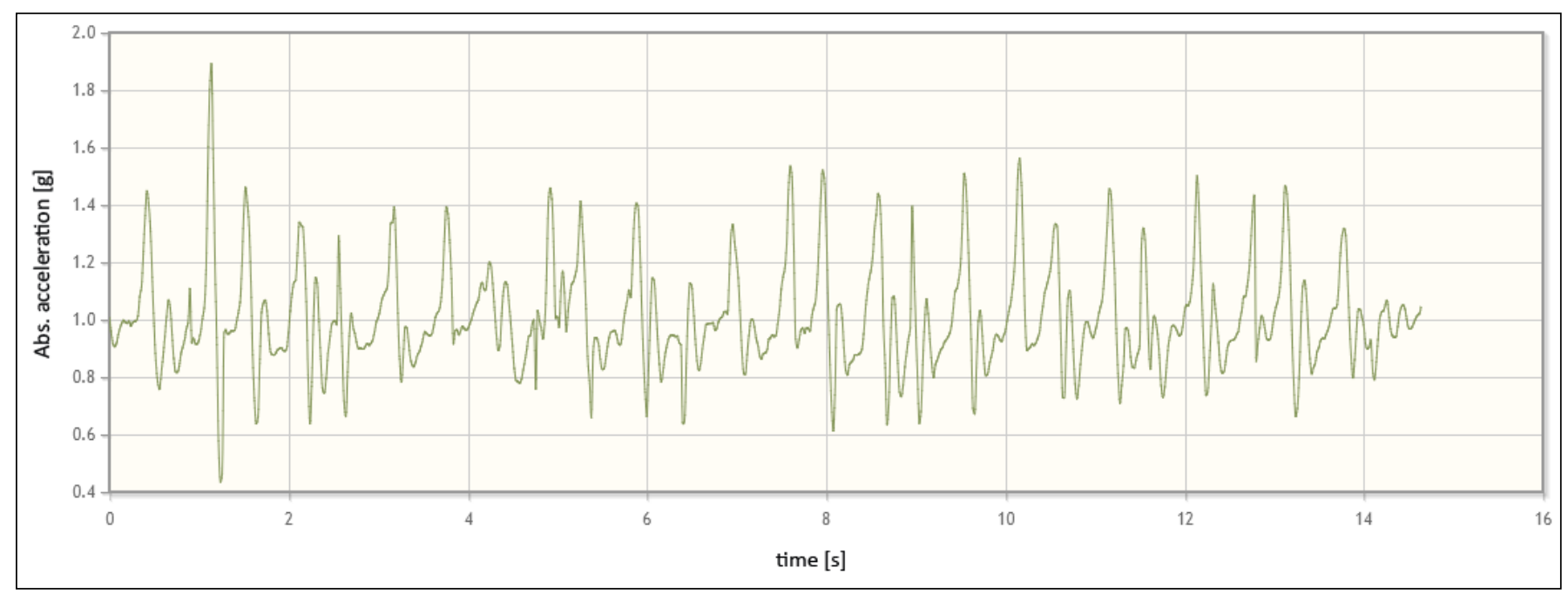

Fig. 6.: Movement pattern of walking on street

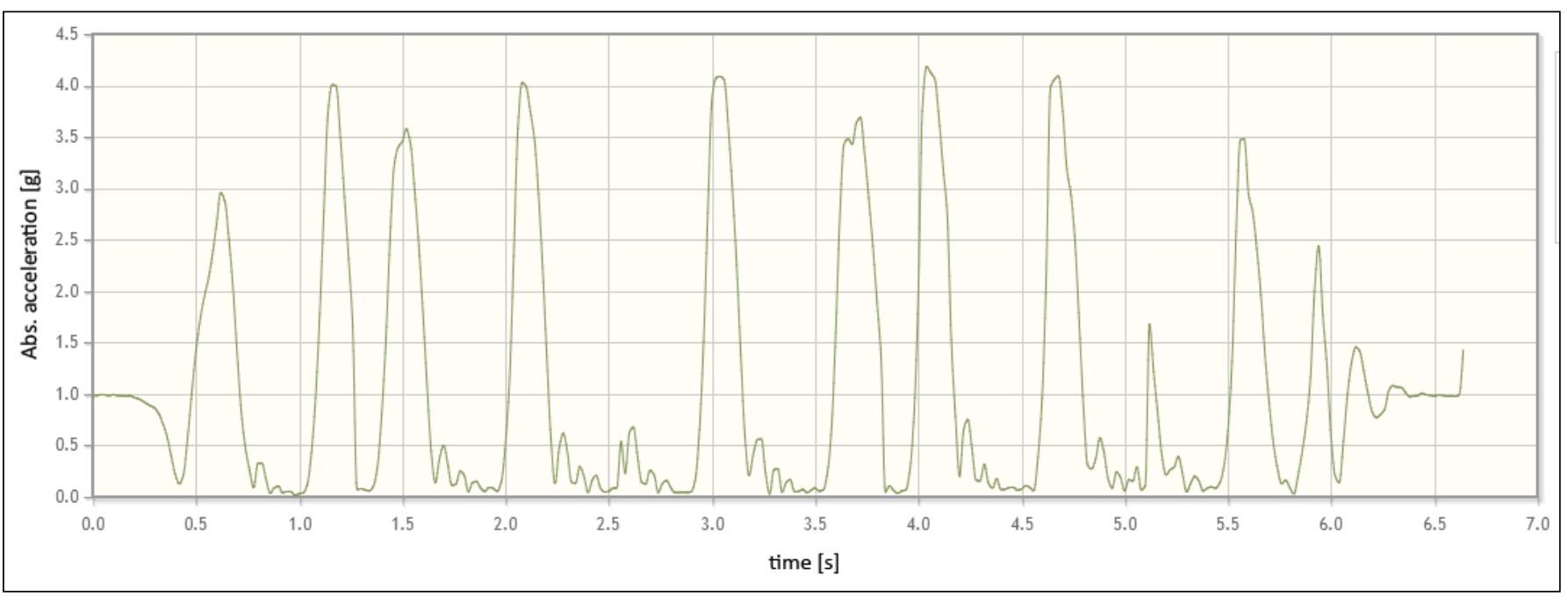

Fig. 7.: Movement pattern of jumping

\section{TECHNICAL IMPLICATIONS}

There are two important points to keep in mind. One is to serve the increased electric power demand came with the acceleration measurements. For this end the previous equipment's power supply had to be further developed. Namely it is not expectable from the wearer to change the batteries frequently, because this would lead to additional costs and inconvenience for the patient. An obvious solution is the rechargeable lithium-cell battery power, however it implies further technical solutions:

- charging the lithium cell and energy management;

- protect the lithium cell against over-charge and under-discharge;

- protect the lithium cell against over-heat;
- monitoring the discharge, the time left to disorder, and intervention of the software in time;

- designing the necessary mechanical connection for recharge.

For these problems the nowadays available, integrated charger circuits provides a lot of help, however in order to monitor the warming, we must choose such a lithium cell, that has an integrated thermistor, and we have to lead it to the microcontroller through an operational amplifier based driver circuit. As long as the temperature reaches a given level, the charging or the consumption must be aborted immediately. It is advisable to choose a cell, which has a built-in circuit which provides protection against overcurrent load and switches the poles of the cell to disconnection over a certain load current. Most of the lithium cells available today includes this security feature. Mechanical design of the charger solved by gold-plated sliding contacts, so we can simply dock the mobile device to the terminal. 
Another consideration is to ensure better co-movement of the equipment with the human body. Not relevant, for instance, an acceleration measurement with a neck strep, dangling instrument, because in this case the equipment does not follows the centre of mass of the individual. For this purpose such an evolving is reasonable which is smaller, lighter and can be fit to the body more easily. For this end the next generation equipment - which is under design at the moment - is:

- integrated into the chest electrode belt;

- smaller and lighter than its predecessor.

\section{RESULTS}

The solutions sketched in the paper result such an equipment form, that supports the comfortable, long-term wearing, and contributes to more accurate and more relevant measurements. The first generation of equipment has also provided many valuable measurement data in our research, but the physical activity is an important compensating factor, to which this degree of modification is reasonable. Taking the activation values into consideration at the evaluation of the collected data is under elaboration, but tests to date indicate great improvement in the usefulness of the measurement data, so this solution is an adequate and proper way of noise-filtering.

\section{ACKNOWLEDGEMENT}

The results discussed above are supported by the grant TÁMOP-4.2.2.B-10/1--2010-0009 and ICI INTERAKTIV Co. Ltd. and Meteo Klinika Ltd.

\section{REFERENCES}

[1] Adam Pinter, Krisztian Samu - Development of a complex microenvironment- and heart monitoring equipment for measuring the effects of the weather on human organism, CERiS'13, Pages 176180, 2013, ISBN 978-963-313-086-5

[2] S.L. Molloy, S. Mihaltcheva - ExtremeWeather Events, Reference Module in Earth Systems and Environmental Sciences, Volume 1: Vulnerability of Human Health to Climate, 2013, Pages 3-16.

[3] R Julian Preston - Uncertainties in estimating health risks associated with exposure to ionising radiation, Journal of Radiological Protection, 2013.

[4] João Vasconcelos - The impact of winter cold weather on acute myocardial infarctions in Portugal, Environmental Pollution, Volume 183, December 2013, Pages 14-18.

[5] S.L. Molloy - Extreme Weather Events and Human Health, International Encyclopedia of Public Health, 2008, Pages 536-544.

[6] Dr Shakoor, Hajat, PhD, Madeline O'Connor, MD, Tom Kosatsky, MD - Health effects of hot weather: from awareness of risk factors to effective health protection, THE LANCET, Volume 375, Issue 9717, 6-12 March 2010, Pages 856-863.

[7] M. S. Islam - An approach to exploring the effect of weather variations on chronic disease incidence rate and potential changes in future health systems, 2010 IEEE 23rd International Symposium on Computer-Based Medical Systems, October 12-October 15.

[8] Jonathan A. Patz, David Engelberg, John Last - The Effects of Changing Weather on Public Health, Annual Review of Public Health, Vol. 21: 271-307, May, 2000.

[9] Alfésio L F Braga - The effect of weather on respiratory and cardiovascular deads in U.S. cities, Environ Health Perspect. 2002 September; 110(9): 859-863.

[10] Vipul Lugade - Validity of using tri-axial accelerometers to measure human movement - Part I: Posture and movement detection, Medical Engineering \& Physics, Volume 36, Issue 2, February 2014, Pages 169-176

[11] Tamar Flash - Models of human movement: Trajectory planning and inverse kinematics studies, Robotics and Autonomous Systems, Volume 61, Issue 4, April 2013, Pages 330-339

[12] Merryn J Mathie - Accelerometry: providing an integrated, practical method for long-term, ambulatory monitoring of human movement, Physiological Measurement, Volume 25, Number 2, 2004

[13] Bobick, A.F. - The recognition of human movement using temporal templates, Browse Journals \& Magazines, Pattern Analysis and Machine, Volume:23 Issue:3, 2001 Pages 257 - 267, ISSN: 01628828

[14] Huba Antal, PhD, Gabor Halasz, PhD - Technical Measurements, ISBN 963420744 8, Pages 29-30.

[15] Gyorgy Lipovszki, PhD - Mechatronics II. education material, 2008, pp. 9-10; Budapest University of Technology and Economics. 